\title{
MEDIDAS DE TENSÃO SUPERFICIAL PELO MÉTODO DE CONTAGEM DE GOTAS: DESCRIÇÃO DO MÉTODO E EXPERIMENTOS COM TENSOATIVOS NÃO-IÔNICOS ETOXILADOS
}

\author{
Érico Teixeira Neto* \\ Centro de Ciências Naturais e Humanas, Universidade Federal do ABC, Rua Santa Adélia, 166, 09210-170 Santo André - SP, \\ Brasil \\ Marcos Maziero Malta \\ Gerência de Desenvolvimento e Aplicação, Oxiteno S.A. Indústria e Comércio, Av. das Indústrias, 365, 09380-903 Mauá - SP, \\ Brasil \\ Ronaldo Gonçalves dos Santos
}

Faculdade de Engenharia Química, Universidade Estadual de Campinas, Cidade Universitária “Zeferino Vaz”, CP 6066, 13083-970 Campinas - SP, Brasil

Recebido em 11/2/08; aceito em 7/7/08; publicado na web em 10/12/08

\begin{abstract}
SURFACE TENSION MEASUREMENT BY DROP COUNTING METHOD: METHOD DESCRIPTION AND EXPERIMENTS WITH ETOXILATED NON-IONIC SURFACTANTS. Surface tension knowledge of surfactants aqueous solutions is important during amphiphilic molecule manufacturing and new product development, as feedback information to handle synthesis parameters to target performance. Drop counting method is an interesting simplification of drop weight method for surface tension measurements. A simple laboratory measurement device, with capability for temperature control, was assembled to allow investigation of ethoxylated surfactants. The implementation of the method was preceded by a detailed investigation of two factors that may affect the measured surface tension: drop formation velocity and surfactant ethoxylation degree. The limitations of the method are discussed on this basis.
\end{abstract}

Keywords: nonylphenol ethoxylates; ethylene oxide; petrochemical industry.

\section{INTRODUÇÃO}

O conhecimento e o controle da tensão superficial de soluções aquosas de tensoativos são fundamentais em diversas aplicações de produtos industrializados. ${ }^{1}$ A adição de pequenas quantidades de tensoativos a diferentes tipos de formulações causa mudanças no comportamento físico-químico dos produtos, permitindo a obtenção de propriedades como, entre outras, maior molhabilidade, melhor dispersão de partículas e maior poder de limpeza. A determinação da tensão superficial de formulações tensoativas é feita rotineiramente em laboratórios de controle de qualidade e de pesquisa industriais, dando suporte ao desenvolvimento de novos produtos e de novas aplicações para os tensoativos.

Na literatura, Barcellos e colaboradores ${ }^{2}$ propuseram uma adaptação simplificada do método do peso da gota para medidas de tensão superficial de soluções de tensoativos. Os autores chamam a atenção para alguns fatores que podem causar erros nas medidas de tensão superficial e que, portanto, devem ser cuidadosamente monitorados durante a realização dos experimentos. Entre eles estão a temperatura das amostras, a velocidade de formação das gotas e o formato da ponta da bureta onde são formadas as gotas. Levando em consideração estes fatores, este trabalho descreve uma nova abordagem de adaptação do método do peso da gota.

A Associação Brasileira de Normas Técnicas (ABNT) prescreve um procedimento ${ }^{3}$ bastante simples e razoavelmente exato para a determinação da tensão superficial de produtos agrotóxicos na NBR 13241. Este procedimento se baseia na contagem do número de gotas gerado por um determinado volume de uma solução aquosa da amostra, medido a partir de uma bureta, e sua relação com o número de gotas gerado pelo mesmo volume de água, que é usada como padrão

*e-mail: erico.teixeira@ufabc.edu.br de tensão superficial conhecida. O método de contagem de gotas pode ser aplicado à determinação da tensão superficial de diferentes tipos de tensoativos, de suas formulações e também de líquidos puros; ele é usado, devido à sua simplicidade, para determinações exploratórias da tensão superficial de soluções tensoativas em laboratórios de pesquisa industriais e acadêmicos.

O método de contagem de gotas descrito na NBR é uma adaptação adequada e simples do método do peso da gota. ${ }^{4}$ Seguindo a argumentação do método do peso da gota, a força exercida pelo peso de uma gota $(\mathrm{m} \mathrm{g})$ na ponta de uma bureta é máxima no momento exatamente anterior ao seu desprendimento da ponta. Neste momento, o peso da gota é equilibrado pela tensão superficial do líquido $(\gamma)$ multiplicada pelo perímetro $(2 . \pi \mathrm{r})$ da ponta da bureta. Assim, a tensão superficial de um líquido pode ser calculada pela medida da massa (m) de uma gota deste líquido, de acordo com a Equação 1:

$\gamma=\frac{m \cdot g}{2 \pi \cdot r}$

Seguindo a Equação 1, também se pode relacionar a massa da $\operatorname{gota}^{5}(\mathrm{~m})$ com o seu volume (V) e a densidade do líquido $(\rho)$, na Equação 2:

$\gamma=\frac{m \cdot g}{2 \pi \cdot r}=\frac{V \cdot \rho \cdot g}{2 \pi \cdot r}$

No método de contagem de gotas, calcula-se a tensão superficial fazendo-se uma relação entre o número de gotas geradas por um volume fixo da amostra, medido em uma bureta, e o número de gotas gerado pelo mesmo volume de água. No sistema descrito neste trabalho, o volume adotado foi de $2 \mathrm{~mL}$, medido em uma pipeta graduada. Assim, nas Equações 3 e 4, estão as relações para 
determinação da massa média de uma gota da amostra e da água, a partir das suas densidades:

$m_{\text {gota }_{\text {AMOSTRA }}}=\frac{2 m L \cdot \rho_{\text {AMOSTRA }}}{n^{\circ} \text { gotas }_{\text {AMOSTRA }}}$

$m_{\text {gota }_{\mathrm{H}_{2} \mathrm{O}} \mathrm{O}}=\frac{2 m L \cdot \rho_{\mathrm{H}_{2} \mathrm{O}}}{n^{\circ} \text { gotas }_{\mathrm{H}_{2} \mathrm{O}}}$

O método proposto na NBR é válido apenas para medidas de tensão superficial de soluções diluídas de tensoativos (até $1 \% \mathrm{~m} / \mathrm{m}$ ). Assim, a Norma assume a densidade da amostra ( $\left.\rho_{\text {AMOSTRA }}\right)$ igual à densidade da água $\left(\rho_{\mathrm{H}_{2} \mathrm{O}}\right)$. Com isso temos:

$m_{\text {gota }_{H_{2}} \mathrm{O}} \cdot n^{\circ}$ gotas $_{\mathrm{H}_{2} \mathrm{O}}=m_{\text {gota }_{\text {AMOSTRA }}} \cdot n^{\circ}$ gotas $_{\text {AMOSTRA }}$

então,

$m_{\text {gota }_{\text {AMOSTRA }}}=\frac{m_{\text {gota }_{\mathrm{H}_{2} \mathrm{O}}} \cdot n^{\circ} \text { gotas }_{\mathrm{H}_{2} \mathrm{O}}}{n^{\circ} \text { gotas }_{\text {AMOSTRA }}}$

A partir da Equação 1:

$\gamma_{\mathrm{H}_{2} \mathrm{O}}=\frac{m_{\text {gotaH }_{2} \mathrm{O}} \cdot g}{2 \pi \cdot r}$ e $\gamma_{\text {AMOSTRA }}=\frac{m_{\text {gota }_{\text {AMOSTRA }}} \cdot g}{2 \pi \cdot r}$

Como a contagem de gotas é feita a partir de uma pipeta, a circunferência e formato da sua ponta são iguais para a amostra e para a água. A partir das equações acima, podemos então fazer a relação abaixo, que resulta na Equação 6.

$$
\begin{gathered}
\frac{\gamma_{\mathrm{H}_{2} \mathrm{O}}}{m_{\text {gotaH }_{2} \mathrm{O}}}=\frac{\gamma_{\text {AMOSTRA }}}{m_{\text {gota }_{\text {AMOSTRA }}}} \mathrm{e} \\
\gamma_{\text {AMOSTRA }}=\frac{m_{\text {gota }_{\text {AMOSTRA }}} \cdot \gamma_{\mathrm{H}_{2} \mathrm{O}}}{m_{\text {gota }_{2} \mathrm{O}}}
\end{gathered}
$$

Substituindo a $m_{\text {gota AMOSTRA }}$ da Equação 5 na Equação 6, obtém-se a relação que permite o cálculo da tensão superficial da solução da amostra, na Equação 7:

$\gamma_{\text {AMOSTRA }}=\frac{n^{\circ} \operatorname{gotas}_{\mathrm{H}_{2} \mathrm{O}} \cdot \gamma_{\mathrm{H}_{2} \mathrm{O}}}{n^{\circ} \text { gotas }_{\text {AMOSTRA }}}$

A velocidade de formação das gotas durante o experimento de contagem de gotas é determinante para o sucesso e a confiabilidade dos resultados obtidos nas medidas. Diferente do método do peso da gota, ${ }^{6}$ que é uma técnica de equilíbrio e pressupõe uma velocidade infinitamente lenta de formação e desprendimento das gotas, o método de contagem de gotas é um método dinâmico, que envolve a formação de gotas em condições de fluxo constante. Portanto, a correlação entre a velocidade de formação de gotas e a tensão superficial que é medida, para os diferentes tipos de amostras tensoativas, deve ser investigada para a adequada implementação do método em nossos laboratórios.

Os tensoativos não-iônicos etoxilados são usados, entre outras aplicações, como tensoativos industriais nos processos de fabricação de lã e de metais, como emulsificantes para polimerização em emulsão, em detergentes de laboratório e em formulações pesticidas. ${ }^{7}$ Eles têm seu grupo hidrofílico formado por uma cadeia oligomérica de óxido de etileno e sua parte hidrofóbica formada por um entre diferentes tipos de cadeias carbônicas, incluindo cadeias alquílicas lineares, ramificadas, insaturadas e cadeias contendo grupos aromáticos, como o nonilfenol. A fórmula geral dos nonilfenóis etoxilados é $\mathrm{C}_{9} \mathrm{H}_{19}-\mathrm{C}_{6} \mathrm{H}_{6}\left(-\mathrm{O}-\mathrm{CH}_{2}-\mathrm{CH}_{2}-\right)_{\mathrm{n}}-\mathrm{OH}$, onde o número $n$ de unidades de óxido de etileno na cadeia da molécula define o seu grau de etoxilação (EO). Uma característica do comportamento de soluções aquosas de tensoativos etoxilados é a marcante dependência de suas propriedades físico-químicas com a temperatura: ${ }^{8}$ a água deixa de ser um bom solvente para os grupos óxido de etileno à medida que se aumenta a temperatura. Portanto, atendendo a esta necessidade, um sistema termostatizado foi construído em nosso laboratório para as determinações de tensão superficial de soluções de tensoativos etoxilados, seguindo o método proposto na NBR 13241.

Este trabalho descreve o sistema termostatizado construído e a metodologia para realização de medidas de tensão superficial de soluções tensoativos etoxilados pelo método de contagem de gotas. O seu objetivo é avaliar e discutir as influências da taxa de formação das gotas e do grau de etoxilação das moléculas tensoativas sobre as tensões medidas, identificando as limitações do método proposto.

\section{PARTE EXPERIMENTAL}

O diagrama esquemático do tensiômetro de contagem de gotas que foi montado nos laboratórios de Pesquisa Analítica da Oxiteno para a realização de medidas de tensão superficial de soluções de tensoativos etoxilados está mostrado na Figura 1. Ele consiste de uma pipeta graduada de $2 \mathrm{~mL}$ que foi fixada por pressão, com rolhas de silicone, em uma camisa termostatizada. Esta foi fabricada usando-se um condensador reto, por onde circula o líquido de termostatização em um banho termostático, com controle de temperatura de $\pm 0,2$ ${ }^{\circ} \mathrm{C}$. A ponta da pipeta foi mantida a uma distância menor que $1,5 \mathrm{~cm}$ da parte inferior do sistema, para garantir a sua termostatização. Um aspirador foi conectado à parte superior da pipeta para o controle preciso da taxa de formação das gotas da solução sob investigação.

As medidas de tensão superficial foram feitas pelo procedimento a seguir: um excesso de água padrão Mili-Q contida em um béquer foi aspirado pela pipeta e mantido em repouso por $1 \mathrm{~min}$ para sua termostatização a $20{ }^{\circ} \mathrm{C}$. O menisco foi então ajustado em $2 \mathrm{~mL}$ e o número de gotas gerado, a uma taxa de formação de gotas de 30 gotas. $\mathrm{min}^{-1}$, foi anotado. A pipeta foi lavada com a amostra, aspirando

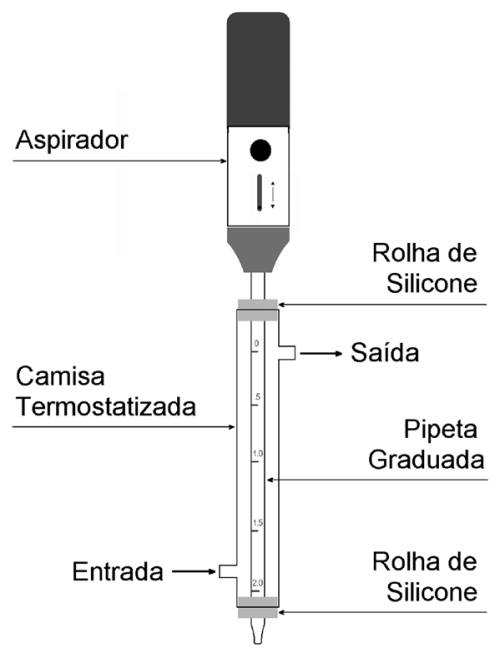

Figura 1. Diagrama esquemático do tensiômetro de contagem de gotas montado nos laboratórios de Pesquisa Analítica da Oxiteno. A termostatização adequada do sistema permite a determinação da tensão superficial dos tensoativos não-iônicos etoxilados 
e descartando-a, por 3 vezes e o procedimento de medida adotado para água foi repetido com a amostra. A tensão superficial da amostra foi calculada usando-se os números de gotas na Equação 7, a média obtida em três repetições do experimento. Também foram realizados experimentos com taxas de formação de gotas de 6 e 3 gotas min $^{-1}$.

Os tensiômetros modelo OCA 15 da DataPhysics (método de gota pendente), disponíveis nos laboratórios de pesquisa da Oxiteno e modelo Sigma 701 da KSV (método de anel de Du Noüy, utilizando a correção matemática de Huh e Mason), ${ }^{9}$ utilizado no Instituto de Química da Unicamp foram usados nas medidas.

Os tensoativos Ultranex NP, uma linha de nonilfenóis etoxilados com diferentes graus de etoxilação produzidos pela Oxiteno, usados nos experimentos estão identificados na Tabela 1. As soluções aquosas dos tensoativos foram preparadas misturando-os com água deionizada padrão Mili-Q a $20{ }^{\circ} \mathrm{C}$ em uma concentração de $0,5 \%$ $(\mathrm{m} / \mathrm{m})$. As soluções foram deixadas em repouso durante $1 \mathrm{~h}$ à mesma temperatura para a realização das medidas.

Tabela 1. Tensoativos nonilfenóis etoxilados investigados, seus respectivos graus de etoxilação, HLB's e massas molares

\begin{tabular}{lccc}
\hline Tensoativo & $\begin{array}{c}\text { Grau de } \\
\text { Etoxilação }\end{array}$ & HLB & $\begin{array}{c}\text { Massa Molar } \\
\left(\mathrm{g} \mathrm{mol}^{-1}\right)\end{array}$ \\
\hline Ultranex NP 50 & 5 & 10 & 441 \\
Ultranex NP 100 & 10 & 13 & 661 \\
Ultranex NP 500 & 50 & 18 & 2423 \\
Ultranex NP 1000 & 100 & 19 & 4625 \\
\hline
\end{tabular}

\section{RESULTADOS E DISCUSSÃO}

A influência da velocidade de formação das gotas sobre a tensão superficial medida no tensiômetro de contagem de gotas foi investigada fazendo-se medidas de uma solução aquosa $0,5 \%(\mathrm{~m} / \mathrm{m})$ de Ultranex NP 500, a $20^{\circ} \mathrm{C}$, com taxas de formação de gotas de 30 , 6 e 3 gotas $\min ^{-1}$. Os resultados da Tabela 2 mostram que a tensão superficial é maior para as mais altas taxas de formação de gotas empregadas no experimento. Este comportamento é marcante: em relação ao experimento feito com 3 gotas $\min ^{-1}$ observou-se um aumento de $3 \mathrm{mN} \mathrm{m}^{-1}$ na tensão superficial quando a taxa de formação de gotas foi de 30 gotas $\mathrm{min}^{-1}$.

Tabela 2. Tensões superficiais ${ }^{a}$ de solução aquosa $0,5 \%(\mathrm{~m} / \mathrm{m})$ de Ultranex NP 500 medidas em diferentes taxas de formação de gotas

\begin{tabular}{cc}
\hline $\begin{array}{l}\text { Taxa de Formação de Gotas } \\
\left(\text { gotas } \min ^{-1}\right)\end{array}$ & Tensão Superficial $\left(\mathrm{mN} \mathrm{m}^{-1}\right)$ \\
\hline 30 & 49 \\
6 & 48 \\
3 & 46 \\
\hline
\end{tabular}

${ }^{a}$ Os erros das medidas estão dentro de $\pm 1 \mathrm{mN} \mathrm{m}^{-1}$, estimados em três replicatas dos experimentos.

Em um trabalho de Jho e Burke, ${ }^{10}$ a mesma correlação entre a tensão superficial medida e a taxa de formação de gotas de soluções de tensoativos foi observada. Atribuíram esse comportamento a fatores hidrodinâmicos, que podem afetar a massa da gota sob condições de fluxo. Segundo eles, o pescoço líquido formado entre a ponta da pipeta e a gota, no momento do seu desprendimento, é empurrado para baixo pelo líquido em fluxo nas taxas mais altas de formação de gotas, o que tende a aumentar a massa da gota e a tensão superficial que é medida. Em trabalho anterior, Pierson e Whitaker ${ }^{11}$ fizeram observações semelhantes, e a mesma argumentação foi usada para justificar o aumento da tensão superficial medida em experimentos com taxa de formação de gotas mais altas que 6 gotas $\min ^{-1}$. A influência da cinética de difusão das moléculas de tensoativo para a superfície da gota, sobre a tensão superficial medida, não foi considerada em ambos trabalhos.

Para examinar mais a fundo o fenômeno de formação das gotas, a dinâmica de relaxação da gota foi investigada sem a influência de fatores hidrodinâmicos. A mesma solução de Ultranex NP 500 (Tabela 2) teve sua tensão superficial dinâmica medida em um tensiômetro de gota pendente. Neste experimento, uma gota da solução é gerada na ponta da pipeta do instrumento e sua tensão superficial é calculada a partir do seu formato, que é registrado fotografando-se a gota ao longo do seu tempo de envelhecimento. Nas fotografias sobrepostas da Figura 2, estão indicados o contorno (preto) da gota de solução de Ultranex NP 500 logo que ela foi gerada $(\mathrm{T}=0 \mathrm{~s}$ ) e a fotografia do perfil (cinza) da mesma gota após 300 s. Pode-se observar que houve uma mudança no formato da gota: ela ficou mais afilada e alongada com seu envelhecimento por $5 \mathrm{~min}$. O formato das gotas é determinado por um balanço entre sua tensão superficial e seu peso. ${ }^{12} \mathrm{Na}$ Figura 2 , $\log$ o que foi formada ( $\mathrm{T}=0 \mathrm{~s}$ ), a gota tinha tensão superficial de $48,7 \mathrm{mN}$ $\mathrm{m}^{-1} \mathrm{e}$, após $300 \mathrm{~s}$, sua tensão diminuiu para $45,2 \mathrm{mN} \mathrm{m}^{-1}$, enquanto o seu volume e, portanto, o seu peso, foram mantidos constantes. Essa variação na tensão superficial é denominada tensão superficial dinâmica e é causada pelo tempo de difusão das moléculas de tensoativo até a superfície da gota recém-formada, onde se organizam em uma monocamada, reduzindo a tensão superficial da gota.

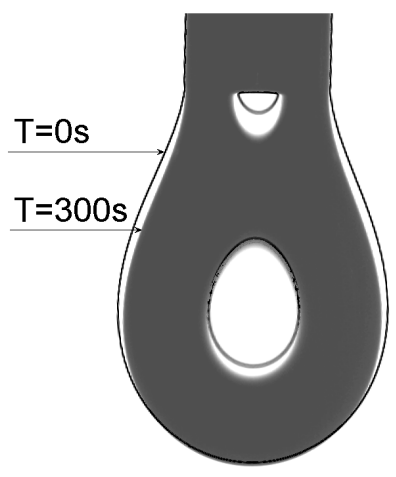

Figura 2. Imagens sobrepostas, obtidas em tempos diferentes, do perfil de uma gota de solução aquosa $0,5 \%(\mathrm{~m} / \mathrm{m})$ de Ultranex NP 500 na ponta da pipeta do tensiômetro de gota pendente. Assim que é gerada $(T=0 \mathrm{~s})$, a gota tem o formato mostrado pelo contorno preto. Após $300 \mathrm{~s}$, seu formato fica mais alongado e afilado (perfil cinza)

Dessa forma, a tensão superficial dinâmica também influencia as medidas de tensão superficial por contagem de gotas, já que o comportamento dinâmico descrito acima se repete a cada gota que é formada. Assim, quanto mais alta é a velocidade de formação de gotas, menor é o tempo de envelhecimento da superfície da gota e, conseqüentemente, mais alta é a tensão superficial das gotas que são geradas.

Nos resultados da Tabela 2, observa-se que, mesmo fazendo-se a medida na menor taxa de formação de gotas avaliada, a tensão medida por contagem de gotas é mais alta que a medida obtida em condições estáticas, por gota pendente após $300 \mathrm{~s}$. Para demonstrar que esse resultado não é causado por efeitos hidrodinâmicos sobre a formação das gotas, foram feitas medidas de tensão superficial de dois líquidos puros, etanol e etilenoglicol, nos quais não se observa tensão superficial dinâmica. Os resultados obtidos considerando-se as densidades dos líquidos puros, foram de, respectivamente, 21 e $47 \mathrm{mN} \mathrm{m}^{-1}$ (valores de 
referência: ${ }^{13} 22,3$ e 48,9 $\mathrm{mN} \mathrm{m}^{-1}$ ) e não foram obtidas diferenças entre as medidas realizadas nas taxas de formação de gotas de 3, 6 e 30 gotas $\min ^{-1}$. No experimento de contagem de gotas, a determinação da tensão superficial de uma amostra é feita à mesma taxa de formação de gotas que a água, que é a referência de tensão superficial conhecida. Assim, os eventuais efeitos hidrodinâmicos, descritos na literatura ${ }^{10,11} \mathrm{em}$ altas taxas de formação de gotas, são aproximadamente compensados.

Podemos então levantar mais uma questão quanto ao uso do tensiômetro de contagem de gotas para a determinação da tensão superficial de soluções de tensoativos etoxilados: como a tensão superficial dinâmica influencia as medidas de tensoativos de uma série homóloga, com diferentes graus de etoxilação?

Primeiramente, a tensão superficial dinâmica de tensoativos nonilfenóis etoxilados, com graus de etoxilação de 5, 10, 50 e 100 EO, foi determinada usando-se o tensiômetro de gota pendente. $\mathrm{Na}$ Tabela 3, estão tabulados os resultados de medidas feitas até $900 \mathrm{~s}$ após a formação da gota. Estes resultados também estão representados na Figura 3A, para visualização e entendimento mais objetivos dos resultados. Comparando-se a tensoatividade (capacidade de diminuir a tensão superficial da solução) das moléculas dessa série homóloga, observa-se, nas tensões superficiais medidas ao final do experimento ( $\mathrm{T}=900 \mathrm{~s}$ ), que os Ultranex NP mais tensoativos são os que têm menor grau de etoxilação. A diminuição no valor da tensão durante o experimento é facilmente observável para os Ultranex NP 500 e NP 1000, mas não é para as moléculas dos NP 50 e NP 100. Na Figura 3B, estão as variações das tensões superficiais medidas com o tempo (d $\gamma / d t)$, das tensões do gráfico da Figura 3A. Observa-se que, durante todo o experimento (até $\mathrm{T}=900 \mathrm{~s}$ ), as soluções dos tensoativos NP 500 e NP 1000 não atingem uma tensão superficial de equilíbrio e a solução do NP 1000 é a que apresenta maior taxa de diminuição da tensão superficial durante praticamente todo o experimento. O tensoativo NP 100 apresenta uma pequena taxa de diminuição da tensão até cerca de $150 \mathrm{~s}$, quando atinge um valor de equilíbrio, e o NP 50 mantém constante sua tensão superficial desde o início do experimento. Dessa forma, pode-se afirmar que a tensão superficial dinâmica é bastante relevante para as moléculas dos tensoativos com maior grau de etoxilação, e maior massa molar, que se difundem mais lentamente dentro da solução em direção à superfície das gotas. Outros fatores também podem contribuir para a tensão superficial dinâmica observada, como a reorientação das moléculas adsorvidas na superfície da gota ${ }^{14}$ e a existência de impurezas tensoativas nas amostras, levando a um equilíbrio de adsorção-dessorção destas impurezas na superfície da gota. Entretanto, a cinética destes processos é muito mais rápida que a cinética do processo de difusão das moléculas etoxiladas e, portanto, sua discussão não agrega à descrição do fenômeno. ${ }^{15}$

A importância ou magnitude da influência da tensão superficial dinâmica sobre as soluções desta série de tensoativos foi determinada fazendo-se medidas de tensão superficial de equilíbrio em um tensiômetro de anel de Du Noüy. Neste método, um anel de platina é submerso em uma solução e a força necessária para se puxar o anel através de sua
A
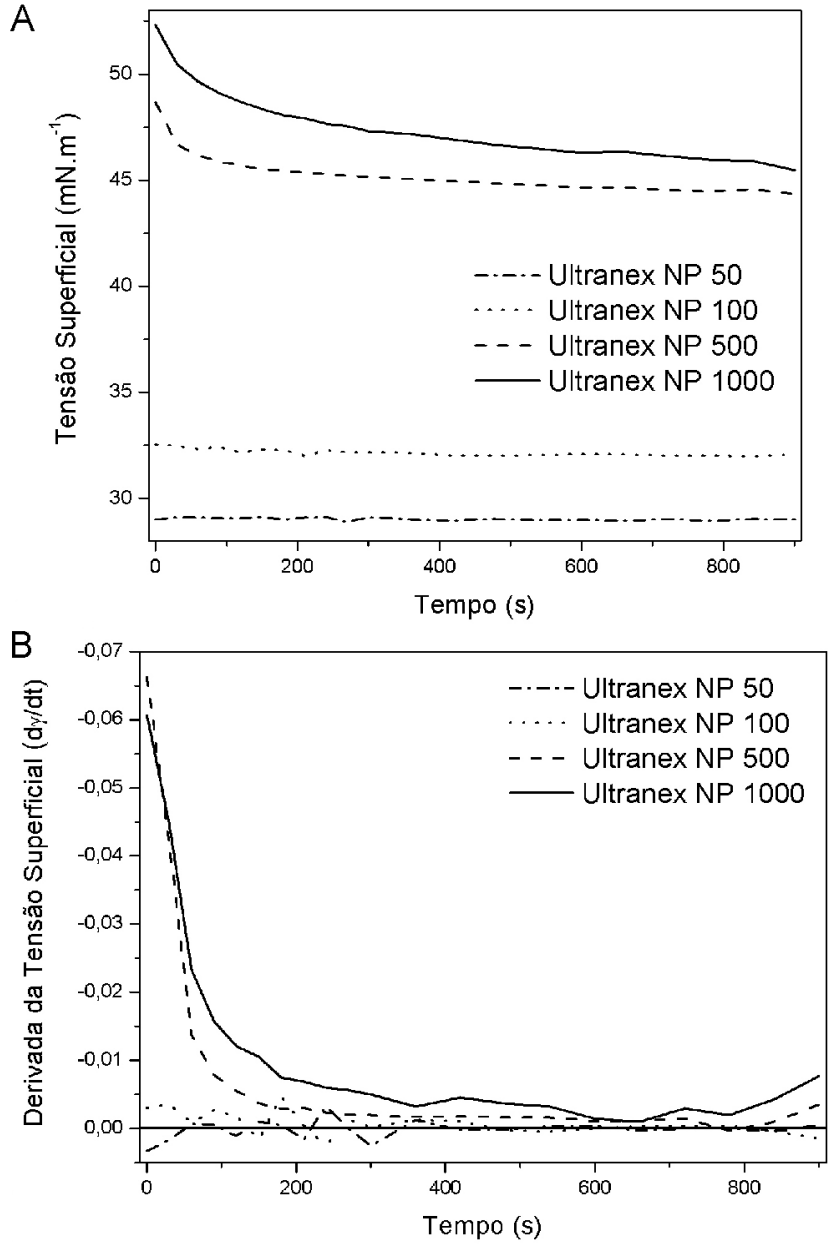

Figura 3. Gráfico (A) com a dinâmica de variação da tensão superficial de soluções aquosas $0,5 \%(\mathrm{~m} / \mathrm{m})$ de tensoativos Ultranex $N P$ medidas após a formação da gota e derivada (B) das tensões medidas no tempo. Em relação aos tensoativos NP 500 e NP 1000, os tensoativos NP 50 e NP 100 causam maior redução na tensão superficial da água e têm dinâmicas mais rápidas de equilíbrio. Na derivada das tensões superficiais com o tempo (B), observa-se que as soluções dos tensoativos NP 500 e NP 1000 não atingem tensão superficial de equilíbrio durante o experimento e a de NP 1000 apresenta maior diminuição da tensão superficial durante praticamente todo o experimento

superfície é medida, permitindo a determinação da tensão superficial em condições de equilíbrio. Os valores obtidos para as soluções dos Ultranex NP investigados encontram-se na Tabela 4, junto com os valores obtidos usando os métodos dinâmicos de contagem de gotas e de gota pendente (em T=900 s), para comparação. Observa-se que os valores de tensão superficial medidos pelo método do anel são menores que os valores

Tabela 3. Tensões superficiais ${ }^{\text {a }}$ de soluções aquosas $0,5 \%(\mathrm{~m} / \mathrm{m})$ de tensoativos Ultranex NP medidas em diferentes tempos após a formação da gota. A diminuição da tensão superficial inicial, com o envelhecimento da gota, é maior para os tensoativos com maior grau de etoxilação

\begin{tabular}{rcccc}
\hline Tensoativos $\rightarrow$ & Ultranex NP 50 & Ultranex NP 100 & Ultranex NP 500 & Ultranex NP 1000 \\
\hline Tempo (s) $\downarrow$ & Tensão $\left(\mathrm{mN} \mathrm{m}^{-1}\right)$ & Tensão $\left(\mathrm{mN} \mathrm{m}^{-1}\right)$ & Tensão $\left(\mathrm{mN} \mathrm{m}^{-1}\right)$ & ${\text { Tensão }\left(\mathrm{mN} \mathrm{m}^{-1}\right)}^{52,3}$ \\
\hline 0 & 29,0 & 32,6 & 48,7 & 47,6 \\
600 & 29,1 & 32,2 & 45,2 & 46,3 \\
900 & 29,0 & 32,1 & 44,7 & 45,5 \\
\hline
\end{tabular}

${ }^{a}$ Os erros das medidas estão dentro de $\pm 0,5 \mathrm{mN} \mathrm{m}^{-1}$, estimados em três replicatas dos experimentos. 
Tabela 4. Tensões superficiais de soluções aquosas $0,5 \%(\mathrm{~m} / \mathrm{m})$ de tensoativos Ultranex NP medidas por diferentes métodos. A diferença entre as tensões medidas pelos diferentes métodos é maior para os tensoativos com maior grau de etoxilação

\begin{tabular}{lcccc}
\hline Tensoativos $\rightarrow$ & Ultranex NP 50 & Ultranex NP 100 & Ultranex NP 500 & Ultranex NP 1000 \\
\hline Método $\downarrow$ & Tensão Medida $\left(\mathrm{mN} \mathrm{m}^{-1}\right)$ & Tensão Medida $\left(\mathrm{mN} \mathrm{m}^{-1}\right)$ & Tensão Medida $\left(\mathrm{mN} \mathrm{m}^{-1}\right)$ & ${\text { Tensão Medida }\left(\mathrm{mN} \mathrm{m}^{-1}\right)}^{\text {Tenas }}$ \\
\hline Contagem de Gotas & 30 & 32 & 49 & 54 \\
Gota Pendente $^{\text {Tensiômetro }}{ }^{\mathrm{a}}$ & 29,0 & 32,1 & 44,4 & 45,5 \\
\hline
\end{tabular}

${ }^{a}$ Os erros das medidas realizadas com o tensiômetro estão dentro de $\pm 0,5 \mathrm{mN} \mathrm{m}^{-1}$, estimados em três replicatas dos experimentos.

medidos pelos outros dois métodos, para todos os tensoativos. Observa-se também que a diferença entre as determinações feitas pelos diferentes métodos cresce com o aumento do grau de etoxilação das moléculas. Estas duas observações demonstram que a tensão superficial dinâmica tem grande influência sobre as soluções dos tensoativos investigados e que o método de contagem de gotas apresenta resultados razoáveis apenas para as moléculas com menor grau de etoxilação (5 e 10 EO).

\section{CONCLUSÃO}

Os limites de uso do método de contagem de gotas para determinação da tensão superficial de soluções aquosas de tensoativos nãoiônicos etoxilados foram investigados para a correta implementação da técnica em nossos laboratórios.

A taxa de formação de gotas influencia diretamente as medidas, resultando em valores até $7 \%$ maiores de tensão superficial para as taxas de formação de gotas mais altas. O grau de etoxilação das moléculas de Ultranex NP também tem influência marcante sobre as medidas. Quanto maior o grau de etoxilação do tensoativo, maior é o tempo necessário para a tensão superficial da gota entrar em equilíbrio. As medidas de tensão superficial obtidas pelo método de contagem de gotas foram até $23 \%$ maiores que as obtidas pelo método do anel de Du Noüy.

O método de contagem de gotas deve então ser usado com cautela na determinação da tensão superficial de soluções tensoativas, pois há uma forte influência da tensão superficial dinâmica sobre as medidas. A metodologia proposta e o sistema termostatizado montado em nosso laboratório mostraram-se adequados para a realização de medidas exploratórias de tensão superficial de tensoativos etoxilados de baixa massa molar e permitirá futuras investigações sobre a influência da temperatura sobre as tensões superficiais de soluções desses tensoativos.

\section{AGRADECIMENTOS}

Os autores agradecem ao Prof. W. Loh pelas valiosas discussões e sugestões durante a elaboração deste trabalho. R. G. dos Santos agradece ao $\mathrm{CNPq}$ pela bolsa de doutoramento.

\section{REFERÊNCIAS}

1. Rangel, R. N.; Colóides: um estudo introdutório, LCTE Editora: São Paulo, 2006, cap. 4.

2. Behring, J. L.; Lucas, M.; Machado, C.; Barcellos, I. O.; Quim. Nova 2004, 27, 492

3. NBR 13241, Agrotóxico - Determinação da tensão superficial; ABNT, 1994.

4. Adamson, A. W.; Physical Chemistry of Surfaces, $5^{\text {th }}$ ed., Wiley-Interscience: New York, 1990, cap. 2; Kaufman, S.; J. Colloid Interface Sci. 1976, 57, 399 .

5. Lando, J. L.; Oakley, H. T.; J. Colloid Interface Sci. 1967, 25, 526.

6. Campbell, J.; J. Phys. D: Appl. Phys. 1970, 3, 1499; Harkins, W. D.; Brown, F. E.; J. Am. Chem. Soc. 1919, 41, 499.

7. http://en.wikipedia.org/wiki/Nonylphenol, acessada em Janeiro 2008.

8. Holmberg, K.; Jönsson, B.; Kronberg, B.; Lindman, B.; Surfactants and Polymers in Aqueous Solution, $2^{\text {nd }}$ ed., John Wiley \& Sons: West Sussex, 2006, cap. 16.

9. Huh, C.; Mason, S. G.; Colloid Polym. Sci. 1975, 253, 566.

10. Jho, C.; Burke, R.; J. Colloid Interface Sci. 1983, 95, 61.

11. Pierson, F. W.; Whitaker, S.; J. Colloid Interface Sci. 1976, 54, 219.

12. Evans, D. F.; Wennerström, H.; The Colloidal Domain - Where Physics, Chemistry, Biology and Technology Meet, $2^{\text {nd }}$ ed., Wiley-VCH: New York, 1999, cap. 2.

13. Azizian, S.; Hemmati, M.; J. Chem. Eng. Data 2003, 48, 662.

14. Ravera, F.; Liggieri, L.; Miller, R.; Colloids Surf. A 2000, 175, 51.

15. Liggieri, L.; Ferrari, M.; Massa, A.; Ravera, F.; Colloids Surf. A 1999, $156,455$. 